\title{
Fuga intrarraquídea de cemento biológico tras cifoplastia. Evolución a largo plazo. Presentación de un caso y revisión de la literatura
}

\author{
J.V. Martínez-Quiñones; J.M. Medina-Imbroda*; J. Aso-Escario; J. Pons Bosque**; F.E. Consolini y R. Arregui- \\ Calvo
}

Servicio de Neurocirugía. Hospital MAZ. Zaragoza. *Servicio de Neurocirugía. Hospital Regional Universitario Carlos Haya. Málaga. ** Servicio de Radiología. Hospital MAZ. Zaragoza.

\section{Resumen}

Introducción. La vertebroplastia y la cifoplastia son técnicas percutáneas mínimamente invasivas indicadas en el tratamiento de ciertas fracturas somáticas vertebrales. Ambas se basan en la introducción dentro del cuerpo vertebral de una sustancia llamada genéricamente cemento, el cual puede ser acrílico o biológico. Son técnicas no exentas de riesgos, destacando como principal complicación la extravasación del cemento. Dada la corta experiencia en el uso de cementos biológicos, existen hoy en día muchas dudas sin resolver acerca de su comportamiento a largo plazo, especialmente en caso de que se produzca una fuga del material de refuerzo vertebral.

Objetivo. Presentamos un caso de extravasación intrarraquídea de cemento biológico durante una cifoplastia en un varón de 23 años y su evolución a largo plazo (3 años), realizando una revisión de la literatura publicada al respecto.

Conclusiones. La mayoría de los estudios publicados tratan sobre la evolución del material de refuerzo dentro de un ambiente óseo, pero no hacen referencia a cómo evolucionaría el mismo fuera del espacio vertebral. Hemos apreciado, en nuestro caso, que el cemento extravasado no está siendo sustituido por hueso, sino que está sufriendo un proceso de reabsorción. La extravasación del material de refuerzo vertebral es una complicación importante y potencialmente grave de las técnicas de refuerzo vertebral percutáneo. Para evitarla se requiere una rigurosa técnica quirúrgica bajo control radioscópico. Los cementos biológicos tienen la ventaja de ser biocompatibles, biodegradables y osteoconductivos. La elección del cemento dependerá de las características de la interacción balón-trabécula.

PALABRAS CLAVE. Vertebroplastia. Cifoplastia. Cemento biológico. Fuga de cemento. Interacción balón-trabécula.

Recibido: 21-12-10. Aceptado: 1-07-11
Biological cement extravasation into the spinal canal following kyphoplasty. Long-term follow-up. Report of one case and review of the literature

Summary

Background. Vertebroplasty and kyphoplasty are minimally invasive percutaneous techniques indicated in the treatment of some somatic vertebral fractures. Both are based on the introduction of a substance, called cement, inside the vertebral body. We can choose acrylic or biological cement. These techniques are not risk-free, cement extravasation being the main complication. Due to the short experience in the use of the biological cements, nowadays there are a lot of unresolved doubts about the long-term behaviour of these materials, especially in the case of leakage.

Purpose. We report a case of biologic cement extravasation into the spinal canal during a kyphoplasty in a 23 year old man and its long-term follow-up (3 years), performing a review of the scientific literature related to the topic.

Conclusions. Most of the papers in the literature discuss the behaviour of the reinforcement material inside a bony environment, but they do not refer to the evolution of the cement outside the vertebral space. We have appreciated in our case that the leaked cement is not being substituted for bone but it suffers a process of pro-

Abreviaturas. $\alpha-1-F P$ : $\alpha$-1-fetoproteina. ACP: fosfato cálcico amorfo. bFGF: factor de crecimiento básico de los fibroblastos. BMP-2: proteina morfogenética del hueso tipo 2. CEA: antígeno carcígeno-embrionario. CP: cifoplastia percutánea. DCPA: fosfato dicálcico anhidroso. DCPD: fosfato dicálcico dihidratado. HA: hidroxiapatita. MCPM: fosfato monocálcico monohidratado. OCP: fosfato octacálcico. PC: fosfato cálcico. PDGF: factor de crecimiento derivado de las plaquetas. PMMA: polimetilmetacrilato. RNM: resonancia nuclear magnética. TC: tomografía computarizada. TCP: fosfato tricálcico. TGF- $\beta$ : factor de crecimiento transformante $\beta$. TTCP: fosfato tetra-cálcico. UH: unidades Hounsfield. VP: vertebroplastia percutánea. 
gressive resorption. The extravasation of the vertebral reinforcement material is an important and potentially serious complication of the vertebral augmentation techniques. To avoid this, a very precise chirurgical technique is necessary, under radiological guidance. Biocompatibility, biodegradability and osteoconductivity are the main advantages of biological cemnets. We base the choice of the cement on the balloom/trabeculae interaction.

KEY WORDS. Vertebroplasty. Kyphoplasty. Biologic cement. Cement leakage. Balloon/trabeculae interaction.

\section{Introducción}

Desde que Jean y Robert Jude ${ }^{21}$ introdujeran el uso de cementos acrílicos de polimetilmetacrilato (PMMA) a principios de los años 40 en la fijación de prótesis parciales de rodilla y posteriormente Charnley extendiera su uso a otras indicaciones ${ }^{7}$, muchas son las aplicaciones que se le han dado a este material en el ámbito de la Cirugía Ortopédica y Traumatológica ${ }^{21}$. Entre estos usos destacan las técnicas de refuerzo vertebral percutáneo: vertebroplastia (VP) y cifoplastia (CP).

La VP fue desarrollada por primera vez en 1987 por Galibert y Deramond ${ }^{15}$ para el tratamiento de angiomas vertebrales sintomáticos, ganando posteriormente gran popularidad como tratamiento de las fracturas vertebrales osteoporóticas y tumorales ${ }^{2,11,19,22,37,45,49}$. A pesar de haber demostrado su eficacia en la mejoría del dolor y la calidad de vida de estos pacientes ${ }^{29,30,33}$, es una técnica que no está exenta de complicaciones, siendo la más frecuente de ellas la extravasación del cemento fuera del cuerpo vertebral $^{11,13,14,16,18,22,46}$.

Con objeto de conseguir, además de la mejoría del dolor y de la calidad de vida, una restauración parcial de la altura normal del cuerpo vertebral, se desarrolló la técnica de CP, llevada a cabo por primera vez por Reiley en $1998^{27}$. Estudios posteriores pusieron de manifiesto que, al reducirse la presión de inoculación del cemento dentro de la cavidad creada por el balón, se reduce de forma significativa el riesgo de extravasación del mismo ${ }^{35,36,44}$.

Los buenos resultados y la evolución de la técnica quirúrgica han hecho que estos procedimientos se extiendan a nuevas indicaciones terapéuticas, como por ejemplo ciertas fracturas vertebrales traumáticas ${ }^{3-5}$. Ésta última ha provocado que dichas técnicas se realicen cada vez en pacientes más jóvenes.

Ahora bien, ¿conocemos cómo se comportan los cementos acrílicos a muy largo plazo? ¿Sabemos cómo puede afectar la presencia de dichos cementos a la propia vértebra y a los niveles adyacentes? En un intento por obviar esta circunstancia, en los últimos años se ha extendido el uso de cementos biológicos derivados del fosfato cálcico en sustitución de los cementos acrílicos, especialmente en pacientes jóvenes ${ }^{3,40}$.

Los primeros trabajos acerca del uso de fosfato cálcico en la reparación de defectos óseos se remontan a principios de los años $20^{1}$. Desde entonces muchos son los estudios que se han realizado con distintos fosfatos de calcio, inicialmente en forma granular mezclados con partículas de cerámica y a partir de mediados de los años 80 en forma de cemento (tabla 1) ${ }^{10}$. Dada la corta experiencia que tenemos con el uso de estos biomateriales en las técnicas de refuerzo vertebral percutáneo, existen hoy en día todavía muchas dudas acerca de su comportamiento a largo plazo. Es más, ¿cómo se comportaría el cemento fuera del cuerpo vertebral en caso de una extravasación del mismo? ¿Se produciría un proceso paulatino de reabsorción o por el contrario sería sustituido por tejido óseo?

Presentamos en este artículo un caso de fuga de cemento biológico en CP y su evolución a largo plazo, realizando posteriormente una revisión de la literatura publicada al respecto.

\section{Caso clínico}

Paciente de 23 años de edad, chapista de profesión, fumador de 20 cigarrillos al día y sin otros antecedentes de interés. En septiembre de 2007 sufre cuadro brusco de sensación de chasquido y dolor en charnela dorso-lumbar mientras saltaba en una cama elástica, motivo por el cual acude al Servicio de Urgencias del Hospital MAZ de Zaragoza, donde, tras estudio radiológico simple, es diagnosticado de una fractura acuñamiento del soma de L2, ingresando posteriormente en el Servicio de Neurocirugía de dicho centro.

$\mathrm{Al}$ ingreso el paciente se encontraba neurológicamente íntegro con dolor intenso a la percusión a nivel de la charnela dorso-lumbar.

Durante su ingreso en planta se realizaron los siguientes estudios complementarios:

- Estudios neurorradiológicos (TC y RNM): fractura somática aguda de L2 sin implicación intrarraquídea.

- Hematimetría y bioquímica sanguíneas: sin hallazgos patológicos.

- Metabolismo fosfo-cálcico: normal.

- Marcadores tumorales (CEA y $\alpha-1-F P)$ : negativos.

- Proteinograma y estudio de inmunoglobulinas: Normales.

- Rastreo gammagráfico óseo (20 mCi - $740 \mathrm{MBq}$ HDP 99mTC): hipercaptación a nivel de L2.

Tras exponer al paciente las distintas opciones terapéuticas, invasivas y no invasivas -reposo en cama y tratamiento ortopédico-, se decide, de común acuerdo con el mismo, 
Tabla 1

Principales trabajos publicados desde 1920 sobre cementos biológicos

\begin{tabular}{|c|c|c|c|}
\hline Año & Autor/es & Tipo de material & Tipo de estudio \\
\hline 1920 & Albee y Morrison & $\mathrm{TCP}+$ cerámica & Experimental (conejos) \\
\hline 1951 & Ray y Ward & $\mathrm{HA}+$ cerámica & Experimental (perros y gatos) \\
\hline 1986 & Brown & $\begin{array}{c}\beta \text {-TCP + cerámica / Cemento } \\
\text { de TTCP + DCPD }\end{array}$ & Ensayo clínico \\
\hline 1991 & Suzuki & DCP vs OCP vs ACP vs HA granulares & Experimental (ratas) \\
\hline 1991-1992 & Costantino y Friedman & Cemento de TTCP + DCPD & Experimental (gatos) \\
\hline 1994 & Bermudez & Cemento de OCP & Experimental (ratas) \\
\hline 1994 & Ito & $\beta$-TCP granular + chitosán & Descriptivo in vitro \\
\hline 1996 & Ito & $\beta$-TCP granular + HA granular + chitosán & Descriptivo in vitro \\
\hline 1996 & Matsuya & $\begin{array}{l}\text { Cemento de TTCP + polímeros de } \\
\text { metilvinileter-ácido maléico }\end{array}$ & Descriptivo in vitro \\
\hline 1996 & Muruyama & HA granular + chitosán & Descriptivo in vitro \\
\hline 1997 & Le Huec & $\beta$-TCP + cerámica & Ensayo clínico \\
\hline 1998 & Chow & Revisión literatura & Revisión \\
\hline 1998 & Yoshikawa & $\begin{array}{l}\text { Cemento de } \alpha-\mathrm{TCP}+\text { ácido cítrico }+ \\
\text { ácido tánico }\end{array}$ & Descriptivo in vitro \\
\hline 1999 & Nakano & Cemento de OCP & Descriptivo in vitro \\
\hline 2000 & Meraw & $\begin{array}{l}\text { Cemento de PC + bFGF + TGF- } \beta+ \\
\text { PDGF + BMP- } 2\end{array}$ & Experimental (perros) \\
\hline 2001 & Doi & $\begin{array}{l}\text { Cemento de TTCP }+\beta-\mathrm{TCP}+\text { ácido } \\
\text { málico/ácido cítrico }\end{array}$ & Comparativo in vitro \\
\hline 2001 & Fujibayashi & $\beta$-TCP $+\mathrm{HA}+$ cerámica & Serie de casos retrospectiva \\
\hline 2001 & Kamakura & OCP granular + cerámica & Experimental (ratas) \\
\hline 2001 & Markovic y Takagi & Cemento de TTCP + DCPA + manitol & Descriptivo in vitro \\
\hline 2001 & Takagi & $\begin{array}{l}\text { Cemento de TTCP + DCPA vs cemento } \\
\text { de PC + bicarbonato sódico }\end{array}$ & Comparativo in vitro \\
\hline 2002 & Yokoyama & Cemento de $\beta$-TCP + TTCP + ácido cítrico & Descriptivo in vitro \\
\hline 2002 & $\mathrm{Xu}$ & Cemento de PC + chitosán & Descriptivo in vitro \\
\hline 2003 & Carey & $\begin{array}{l}\text { Cementos premezclados (MCPM vs PC + } \\
\text { chitosán/ácido tartárico) }\end{array}$ & Comparativo in vitro \\
\hline 2003 & Chow & Cemento ácido de PC vs HA granular & Comparativo in vitro \\
\hline 2003 & Takagi & $\begin{array}{l}\text { Cementos premezclados (TTCP + } \\
\text { DCPA vs } \alpha \text {-TCP vs DCPA) }\end{array}$ & Comparativo in vitro \\
\hline
\end{tabular}




\begin{tabular}{|l|c|c|c|}
\hline Año & Autor/es & Tipo de material & Tipo de estudio \\
\hline 2003 & Takagi & Cemento de TTCP + DCPA + chitosán & Descriptivo in vitro \\
\hline 2004 & Edwards & PC granular + BMP-2 & Experimental (perros) \\
\hline 2004 & Ruhé & Cemento de PC + BMP-2 & Experimental (conejos) \\
\hline 2004 & Sugawara & Cemento de PC + manitol & Experimental (ratas) \\
\hline 2004 & Xu & Cemento de PC + chitosán/fibras de vicril/ambos & Comparativo in vitro \\
\hline 2004 & Cemento de PC + chitosán/ & Comparativo in vitro \\
\hline 2005 & Bohner & Revisión literatura & Revisión \\
\hline 2005 & Kroese-Deutman & Cemento de PC + BMP-2 & Experimental (conejos) \\
\hline 2005 & Kuemmerle & Cemento ácido de PC + brucita & Experimental (ovejas) \\
\hline 2005 & Zhang & Cemento de PC + chitosán/fibras de vicril & Comparativo in vitro \\
\hline 2007 & Sun & Cemento de PC + chitosán & Descriptivo in vitro \\
\hline 2007 & Xu & Cemento de PC + chitosán & Descriptivo in vitro \\
\hline
\end{tabular}

TCP: Fosfato tricálcico. HA: Hidroxiapatita. TTCP: Fosfato tetra-cálcico. DCPD: Fosfato dicálcico dihidratado. OCP: Fosfato octacálcico. ACP: Fosfato cálcico amorfo. PC: Fosfato cálcico. MCPM: Fosfato monocálcico monohidratado. bFGF: Factor de crecimiento básico de los fibroblastos. TGF- $\beta$ : Factor de crecimiento transformante $\beta$. PDGF: Factor de crecimiento derivado de las plaquetas. BMP-2: Proteina morfogenética del hueso tipo 2. DCPA: Fosfato dicálcico anhidroso.

llevar a cabo un refuerzo vertebral percutáneo mediante $\mathrm{CP}$ con cemento biológico KyphOs $\mathrm{FS}^{\mathrm{TM}}$, procedimiento que se llevó a cabo bajo anestesia general a los seis días de su ingreso siguiendo la metodología quirúrgica habitual de Nuestro Centro: enhebrado pedicular bilateral bajo control radioscópico, insuflado progresivo de los balones (apreciándose durante esta fase una resistencia inicial elevada de la trabécula a su deformidad por el balón de cifoplastia, hasta vencerla -punto de rotura-, a partir del cual se consigue una distensión progresiva de los balones sin apenas oposición), remodelado del soma vertebral, y relleno con cemento biológico de los nichos "creados" en el interior del soma vertebral con 2,3 y 2,4 c.c., izquierdo y derecho respectivamente, sin dificultad ${ }^{27}$.

En el postoperatorio inmediato, se apreció que el paciente presentaba una debilidad franca en el cuádriceps derecho con fenómenos disestésicos asociados. Al no apreciar patología añadida en el estudio radiológico simple que se le practicó de urgencia, se sometió al paciente a un estudio de TC, apreciándose una fuga intrarraquídea del material de refuerzo utilizado (figura 1). A las dos horas de la cirugía el paciente inició una mejoría en la movilidad del cuádriceps, por lo que se mantuvo una actitud expectante.
Doce horas más tarde el paciente movilizaba la extremidad sin dificultad, aunque persistían los fenómenos disestésicos por los dermatomas L2 y L3 derechos. A la semana del procedimiento el paciente fue dado de alta hospitalaria con una práctica ausencia de síntomas, manteniéndose controles ambulatorios en Consultas Externas de Neurocirugía. A los tres meses fue dado de alta laboral, mostrándose en ese momento completamente asintomático. Para estudiar la evolución de la fuga intrarraquídea se siguieron controles periódicos cada seis meses hasta cumplir el primer año de seguimiento, siendo posteriormente anuales (figura 2).

\section{Discusión}

Tanto la vertebroplastia como la cifoplastia son técnicas quirúrgicas mínimamente invasivas útiles en el tratamiento de ciertas fracturas somáticas vertebrales. Las principales indicaciones de estos procedimientos en el momento actual $\operatorname{son}^{27}$ :

- Fracturas vertebrales de origen osteoporótico.

- Hemangiomas sintomáticos.

- Metástasis óseas osteolíticas. 
Neurocirugía

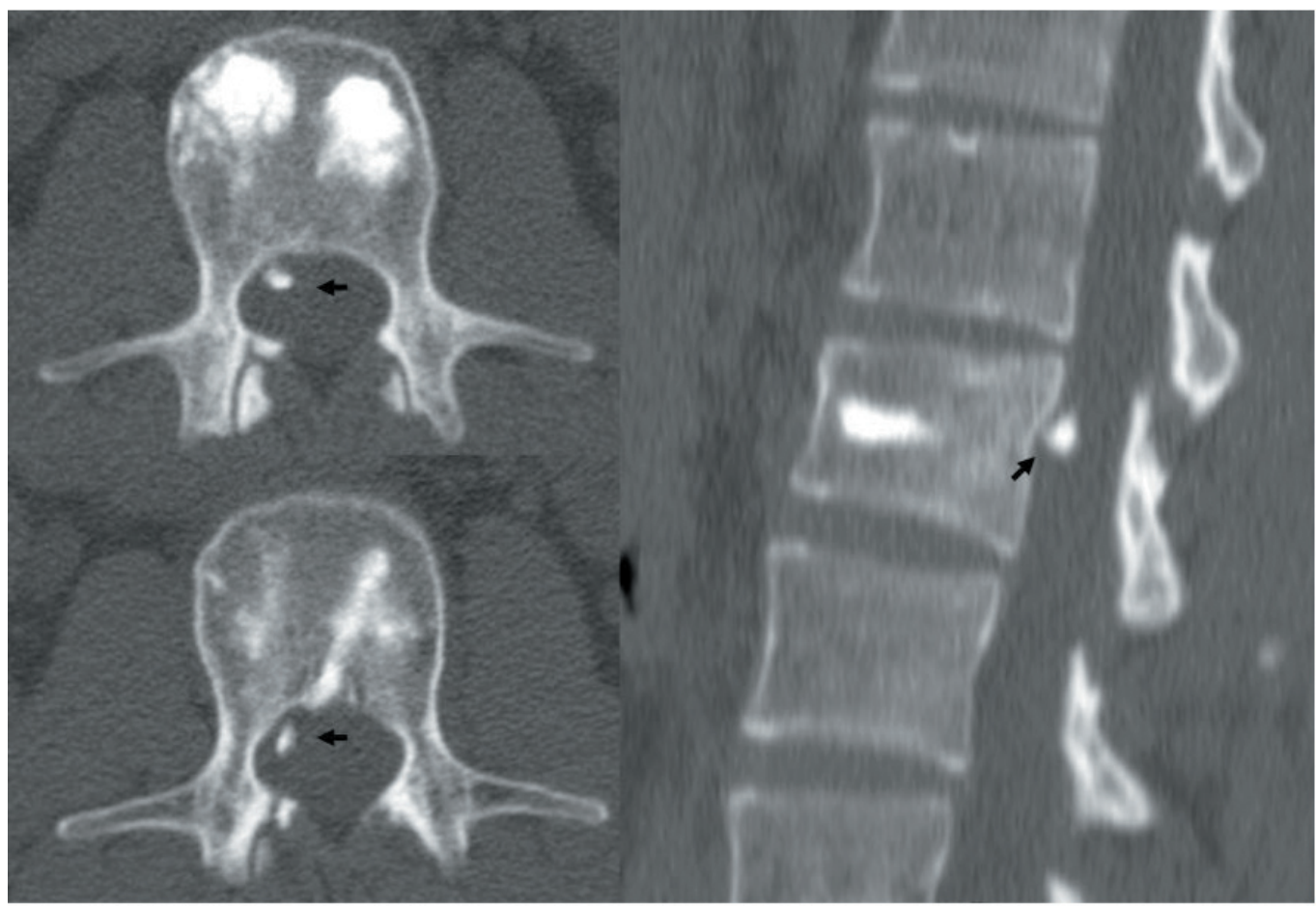

Figura 1. Se aprecia la fuga de material de refuerzo vertebral al espacio epidural a través del agujero nutricio de la vértebra (flecha)

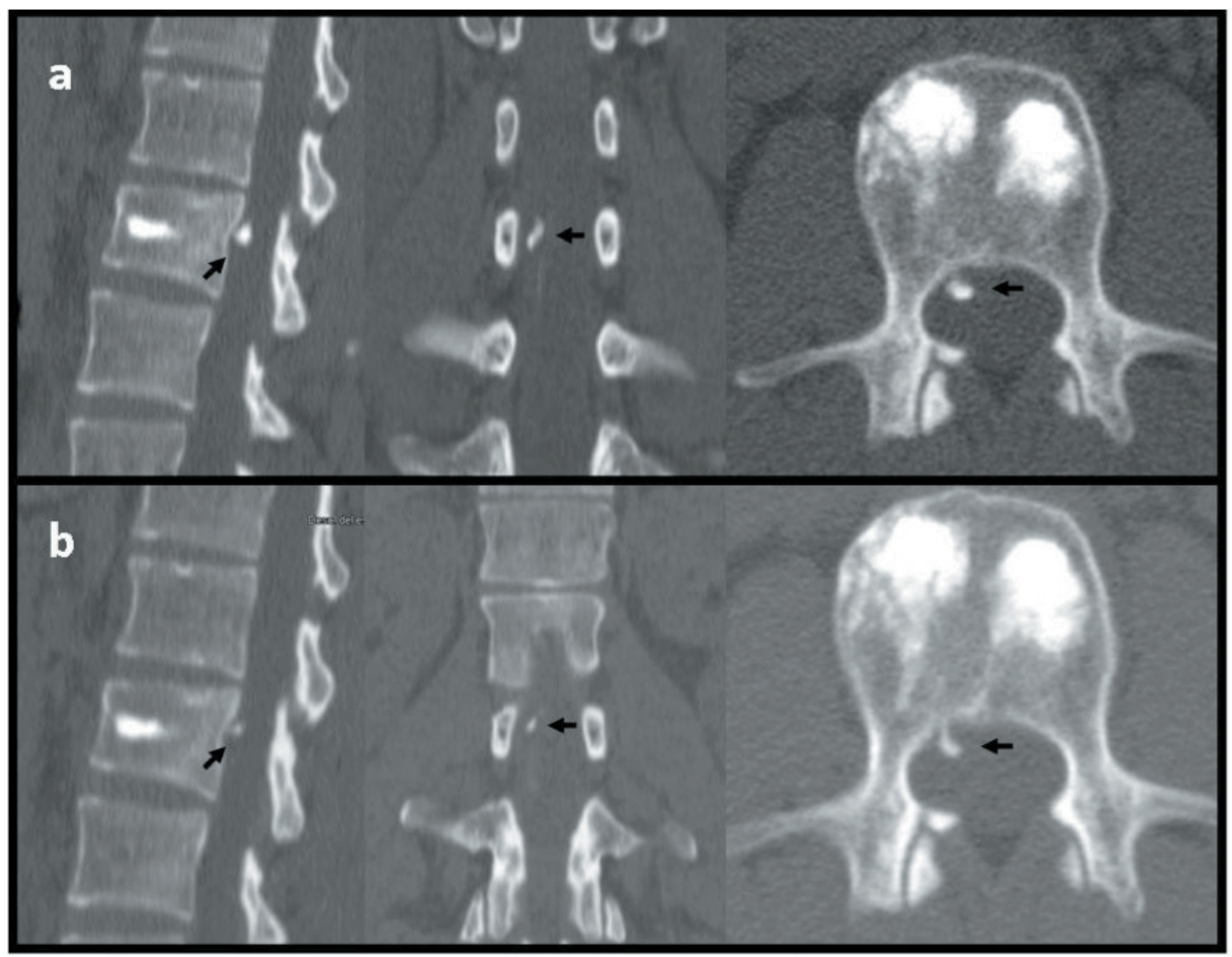

Figura 2. Control evolutivo de la fuga de cemento (flechas). Imagen 2a: Control en diciembre de 2008 sin cambios significativos respecto a la imagen inicial. Imagen 2b: Control en diciembre de 2010 con disminución de tamaño del cemento extravasado. 
- Mieloma múltiple.

- Casos seleccionados de fracturas vertebrales traumáticas.

- Otras:

- Linfoma

- Espondilitis de Kummel

- Histiocitosis

- Osteogénesis imperfecta

Ambos procedimientos se basan en la introducción dentro del soma vertebral de determinadas sustancias, llamadas genéricamente cementos, que tienen capacidad de precipitar en forma de agregados de alta dureza. Estos cementos pueden dividirse en dos tipos: acrílicos y biológicos.

Los cementos acrílicos están constituidos por dos fases, una sólida en forma de polvo y una líquida. El líquido consta fundamentalmente de moléculas de metilmetacrilato (97-99\%), a lo cual se suma un acelerador (N,N-dimetilp-toluidina) y trazas de un estabilizador que previene la polimerización precoz del compuesto (hidroquinona). La composición del polvo puede ser más variable, pero en general consta de microesferas de PMMA u otro polímero que actuarán como núcleos de polimerización (83-99\%) a lo cual se añade una sustancia radioopaca como sales de bario, zinc o estroncio (que no intervienen en la reacción) y un iniciador de la polimerización. La mezcla de todos estos componentes desencadena una reacción exotérmica que conlleva la polimerización y endurecimiento del cemento ${ }^{21}$.

Los cementos biológicos, en su mayoría, también están constituidos por una fase sólida en forma de polvo y un líquido. La composición del polvo es mucho más variable que en el caso de los cementos acrílicos (tabla 2) pero habi- tualmente se compone de una o varias formas de fosfato cálcico a la cual se asocian una serie de sustancias que actúan como estabilizadores, catalizadores y sustancias radioopacas. El líquido se compone de una sustancia que actúa como iniciador. Al mezclar estas dos fases se producen una serie de reacciones químicas que llevan, en la gran mayoría de los casos, a la formación y precipitación de cristales de hidroxiapatita (HA), aunque pueden formarse otras formas de sales de fosfato cálcico ${ }^{10}$.

Por otro lado, los cementos biológicos pueden clasificarse en función del tipo de reacción química predominante que provoca el endurecimiento del mismo, lo cual determina además sus características físicas y biológicas. Así, podemos diferenciar tres tipos de cementos: 1. Aquellos en los que el endurecimiento se debe a la reacción entre distintas formas de fosfato cálcico; 2. Aquellos en los que la reacción fundamental se produce entre un fosfato cálcico y un ácido orgánico; 3 . Aquellos en los que reaccionan un fosfato cálcico con un polímero. Estos mecanismos no son excluyentes entre $\mathrm{si}^{10}$. En la tabla 3 se muestran algunos estudios a cerca de estos cementos y sus conclusiones principales.

Además de estos estudios, existen otros muchos trabajos en los que se añaden diferentes sustancias a los cementos ya citados para modificar o mejorar alguna de sus características $^{10}$. Así se ha comprobado que la incorporación de ciertas fibras como el vicril o el chitosán aumenta la resistencia del cemento resultante ${ }^{47}$. La creación de macroporos en el cemento mediante la incorporación de ciertas sustancias hidrosolubles como el bicarbonato ${ }^{42} \mathrm{O}$ el manitol ${ }^{25}$ aumentan la capacidad de osteointegración a expensas de un descenso en su resistencia. Por otra parte, algunos trabajos han estudiado el efecto osteoinductor que

Tabla 2

Formas de fosfatos cálcicos y sus fórmulas

\begin{tabular}{|c|c|}
\hline Nombre & Fórmula \\
\hline ACP: Fosfato cálcico amorfo & $\mathrm{Ca}_{3}\left(\mathrm{PO}_{4}\right)_{2}$ \\
\hline$\alpha$-TCP: $\alpha$-Fosfato tricálcico & $\alpha-\mathrm{Ca}_{3}\left(\mathrm{PO}_{4}\right)_{2}$ \\
\hline$\beta$-TCP: $\beta$-Fosfato tricálcico & $\beta-\mathrm{Ca}_{3}\left(\mathrm{PO}_{4}\right)_{2}$ \\
\hline DCPA: Fosfato dicálcico anhidroso & $\mathrm{CaHPO}_{4}$ \\
\hline DCPD: Fosfato dicálcico dihidratado & $\mathrm{CaHPO}_{4} \cdot 2 \mathrm{H}_{2} \mathrm{O}$ \\
\hline HA: Hidroxiapatita & $\mathrm{Ca}_{10}\left(\mathrm{PO}_{4}\right)_{6}\left(\mathrm{OH}_{2}\right)_{2}$ \\
\hline MCPA: Fosfato monocálcico anhidroso & $\mathrm{Ca}_{2}\left(\mathrm{H}_{2} \mathrm{PO}_{4}\right)_{2}$ \\
\hline MCPM: Fosfato monocálcico monohidratado & $\mathrm{Ca}_{2}\left(\mathrm{H}_{2} \mathrm{PO}_{4}\right)_{2} \cdot 2 \mathrm{H}_{2} \mathrm{O}$ \\
\hline OCP: Fosfato octacálcico & $\mathrm{Ca}_{8} \mathrm{H}_{2}\left(\mathrm{PO}_{4}\right)_{6} \cdot 5 \mathrm{H}_{2} \mathrm{O}$ \\
\hline TTCP: Fosfato tetra-cálcico & $\mathrm{Ca}_{4}\left(\mathrm{PO}_{4}\right)_{2}{ }^{\circ}$ \\
\hline
\end{tabular}




\section{Tabla 3}

Conclusiones principales de estudios con cementos biológicos Se presentan los compuestos iniciales del cemento y el precipitado final separados por $\rightarrow$

\begin{tabular}{|c|c|c|}
\hline \multicolumn{3}{|c|}{ Reacción entre fosfato cálcico y fosfato cálcico } \\
\hline Autor & Cemento & Conclusiones \\
\hline Constantino y Friedman & $\mathrm{TTCP}+\mathrm{DCPD} \rightarrow \mathrm{HA}$ & $\begin{array}{l}\text { Sustitución gradual de la HA por tejido óseo sin } \\
\text { pérdida de volumen }\end{array}$ \\
\hline Bermudez & $\mathrm{PC}+\mathrm{PC} \rightarrow \mathrm{OCP}$ & $\begin{array}{l}\text { Se puede acelerar el endurecimiento alterando el pH } \\
\text { de la solución. Reabsorción más rápida que la HA }\end{array}$ \\
\hline Bohner & $\alpha-\mathrm{TCP}+\mathrm{MCPM} \rightarrow \mathrm{HA}$ & $\begin{array}{l}\text { Al ser los precursores menos solubles se precisa } \\
\text { catalizador }\end{array}$ \\
\hline Brown & $\mathrm{TTCP}+\mathrm{DCPD} \rightarrow \mathrm{HA}$ & $\begin{array}{l}\text { Al tener los dos precursores más solubles y el } \\
\text { producto final más estable tiene el menor tiempo de } \\
\text { endurecimiento }\end{array}$ \\
\hline Chow & $\mathrm{PC}+\mathrm{PC} \rightarrow \mathrm{DCPD}$ & $\begin{array}{c}\text { Requieren menor pH para endurecer. Menor } \\
\text { resistencia que HA }\end{array}$ \\
\hline Nakano & $\mathrm{PC}+\mathrm{PC} \rightarrow \mathrm{OCP}$ & $\begin{array}{l}\text { Mayor solubilidad del OCP que la HA por lo que } \\
\text { tarda más en endurecerse }\end{array}$ \\
\hline Kuemmerle & $\mathrm{PC}+\mathrm{PC} \rightarrow \mathrm{DCPD}+$ Brucita & Reabsorción más rápida que con HA \\
\hline \multicolumn{3}{|c|}{ Reacción entre fosfato cálcico y ácido orgánico } \\
\hline Autor & Cemento & Conclusiones \\
\hline Doi & $\begin{array}{l}\mathrm{TTCP}+\beta-\mathrm{TCP}+\text { ácido } \\
\text { málico/ácido cítrico } \rightarrow \mathrm{HA}\end{array}$ & $\begin{array}{c}\text { Endurecimiento más rápido. Más fácil de moldear. } \\
\text { Menor resistencia }\end{array}$ \\
\hline Yokoyama & $\beta$-TCP + TTCP + ácido cítrico & $\begin{array}{l}\text { Endurecimiento más rápido. Más fácil de moldear. } \\
\text { Menor resistencia }\end{array}$ \\
\hline Yoshikawa & $\begin{array}{l}\alpha-\mathrm{TCP}+\text { ácido cítrico }+ \\
\text { ácido tánico } \rightarrow \mathrm{HA}\end{array}$ & $\begin{array}{c}\text { Endurecimiento más rápido. Más fácil de moldear. } \\
\text { Menor resistencia }\end{array}$ \\
\hline \multicolumn{3}{|c|}{ Reacción entre fosfato cálcico y polímeros } \\
\hline Autor & Cemento & Conclusiones \\
\hline Matsuya & $\begin{array}{l}\text { TTCP + polímeros de } \\
\text { metilvinileter-ac. maleico }\end{array}$ & $\begin{array}{c}\text { Endurecimiento más rápido que sin polímeros. } \\
\text { Mayor resistencia }\end{array}$ \\
\hline Takagi & $\begin{array}{l}\text { TTCP + DCPA + } \\
\text { Chitosán } \rightarrow \text { HA }\end{array}$ & $\begin{array}{c}\text { Endurecimiento más rápido. Mayor resistencia. } \\
\text { Propiedades elastoméricas }\end{array}$ \\
\hline $\mathrm{Xu}$ & PC + Chitosán & Endurecimiento más rápido. Mayor resistencia \\
\hline
\end{tabular}

TCP: Fosfato tricálcico. HA: Hidroxiapatita. TTCP: Fosfato tetra-cálcico. DCPD: Fosfato dicálcico dihidratado. OCP: Fosfato octacálcico. PC: Fosfato cálcico. MCPM: Fosfato monocálcico monohidratado. 


\section{Tabla 4}

Composición del cemento KyphOs FS ${ }^{\mathrm{TM}}$

\begin{tabular}{|c|c|c|}
\hline \multicolumn{2}{|c|}{ Composición del polvo } & \\
\hline Compuesto & Fórmula & Peso (\%) \\
\hline$\alpha-\mathrm{TCP}$ & $\alpha-\mathrm{Ca}_{3}\left(\mathrm{PO}_{4}\right)_{2}$ & 70.3 \\
\hline Fosfato magnésico & $\mathrm{Mg}_{3}\left(\mathrm{PO}_{4}\right)_{2}$ & 13.0 \\
\hline Fosfato de hidroxi-magnesio & $\mathrm{MgHPO}_{4}$ & 3.3 \\
\hline Carbonato de estroncio & $\mathrm{SrCO}_{3}$ & \\
\hline & $\mathrm{Composición} \mathrm{del} \mathrm{líquido}$ & \multirow{2}{*}{ Ratio polvo/líquido (mg/ml) } \\
\hline Compuesto & Fórmula & 0.4 \\
\hline Fosfato de hidroxi-diamonio & $\left(\mathrm{NH}_{4}\right)_{2} \mathrm{HPO}_{4}$ & \\
\hline TCP: Fosfato tricálcico & & \\
\hline
\end{tabular}

tendría la incorporación a los cementos de ciertos factores de crecimiento $^{31}$. Finalmente, en los últimos años se han desarrollado algunos cementos premezclados que tienen la ventaja de no endurecerse hasta que se inyectan dentro del hueso, pero con la desventaja de que dicho endurecimiento es mucho más lento y su resistencia es menor ${ }^{43}$. (Tabla 1).

El cemento usado en nuestro caso fue un cemento biológico de $\alpha$-TCP que precipita en forma de cristales de HA tras ser mezclado con una disolución de un iniciador (KyphOs FS ${ }^{T M}$. Kyphon Inc. Medtronic Spine LLC. Sunnyvale, $C A, U S A)$. La esterilización del compuesto se realiza mediante radiación $\gamma$. Su composición se muestra en la tabla 4.

Tanto los cementos acrílicos como los biológicos tienen ventajas e inconvenientes. Los cementos acrílicos consiguen una estabilización primaria de la vértebra que es mucho más resistente que en el caso de los biológicos (fuerza de compresión in vitro de unos 50-100 MPa frente a $25 \mathrm{MPa}$ ) y además son más radioopacos, lo cual facilita la detección de extravasación del cemento en tiempo real. Sin embargo este tipo de cementos tiene la desventaja de fraguar a altas temperaturas $\left(70-80^{\circ}\right.$ in vitro y $40-50^{\circ}$ in vivo) y carecer de actividad osteoinductiva u osteointegrativa. Además el PMMA puede ser alergénico, llegando en algunos casos a producir anafilaxia. Por el contrario, los cementos biológicos fraguan a menor temperatura, son biocompatibles y osteoinductivos y se reabsorben, sustituyéndose, en principio, por tejido óseo. Sin embargo, su resistencia es menor, especialmente a las fuerzas de flexión, y son más radiotransparentes ${ }^{21,40}$.

A pesar de las diferencias entre los dos tipos de cementos, no existen en la actualidad estudios clínicos que com- paren la implicación de cada uno de ellos en la aparición de complicaciones tras un procedimiento de refuerzo vertebral percutáneo. Pensamos que esto es debido, fundamentalmente, a la relativa corta experiencia en el uso de cementos biológicos en estas técnicas y a que los grupos de tratamiento no serían comparables, ya que cada tipo de cemento estaría indicado en pacientes de características muy distintas. Las complicaciones más importantes de los procedimientos de refuerzo vertebral reflejadas en la literatura $\operatorname{son}^{23,27}$ :

- Extravasación del cemento

- Fracturas vertebrales en niveles adyacentes

- Otras menos frecuentes:

- Complicaciones derivadas de la extravasación del cemento

- Osificación heterotópica ${ }^{8}$

- Osteitis aséptica de niveles adyacentes ${ }^{48}$

En cuanto a la fractura vertebral adyacente existe cierta controversia al respecto, ya que mientras que ciertos estudios aseguran que el endurecimiento vertebral es responsable directo de la aparición de las mismas, llegando a reportar un riesgo de entre el 10 y el 14\%, dependiendo de las series ${ }^{27}$, en otros estudios se rechaza tal aseveración ${ }^{8,11}$, haciendo responsable de la misma al proceso patológico subyacente a la fractura, que en la mayoría de los casos es la osteoporosis. El resto de las complicaciones son excepcionales y su papel en la literatura es meramente anecdótico.

La extravasación del cemento es la complicación más frecuente y objeto del presente trabajo. El riesgo de extravasación del cemento se encuentra, según las series, entre el $20-72^{\prime} 5 \%$ para la VP y el $1-49^{\prime} 2 \%$ para la CP, 
aunque en la mayoría de las series el riesgo para esta última suele ser menor al $7 \%{ }^{23,27}$. Habitualmente la fuga de cemento suele ser asintomática, aunque, en función del espacio al que se produzca la extravasación, pueden aparecer complicaciones de mayor entidad e incluso mortales ${ }^{23}$.

Las fugas de cemento se pueden dividir en tres tipos en función de la zona por la que sale el mismo ${ }^{28,48}: 1$. Tipo 1 o B, cuando se produce por la vena basivertebral; 2 . Tipo 2 o S, cuando es a través de una vena segmentaria; 3 . Tipo 3 o $\mathrm{C}$, cuando se produce a través de un defecto cortical, ya sea por la propia fractura o por rotura del pedículo en las maniobras quirúrgicas ${ }^{34}$. Por otro lado, podemos separarlas en función al espacio hacia el cual sale el cemento ${ }^{39}$ : al tejido blando perivertebral (40-48\%), al espacio intervertebral (35-38\%) o al canal espinal (5-11\%), siendo más raras otras localizaciones como a través del canal de trabajo del pedículo.

Aunque, como ya se ha comentado, la mayor parte de las veces la extravasación de cemento es asintomática, en algunas ocasiones pueden dar lugar a complicaciones mayores. Así, la salida al espacio intrarraquídeo se ha relacionado con aparición de radiculopatías ${ }^{9}$ e incluso paraparesia ${ }^{34}$, precisándose en algunos casos descompresión quirúrgica urgente. La salida al espacio intervertebral se ha relacionado con dolor persistente y mayor riesgo de fracturas adyacentes $^{8}$. Se han publicado casos de abscesos secundarios a fuga en el tejido blando perivertebral. Finalmente, la salida del cemento a través de los vasos se ha asociado a embolismo pulmonar y, en casos excepcionales, a roturas cardiacas o embolismo arterial ${ }^{23}$.

En nuestro caso la fuga del cemento se produjo a través del agujero nutricio de la vértebra, probablemente por una continuidad de la línea de fractura hacia el mismo, hecho del cual no hemos encontrado otras referencias en la literatura. El cemento se alojó en el espacio intrarraquídeo, dando lugar a una clínica de afectación neurológica inmediata, pero dada la escasa cuantía de la fuga y la recuperación precoz del paciente se decidió adoptar una conducta expectante. Esta fuga pasó desapercibida durante el procedimiento técnico debido a la escasa radio-opacidad que presenta el cemento utilizado durante la fase de introducción y no, como pudiera interpretarse, a una introducción del mismo a elevada presión.

Existen múltiples estudios en la literatura que pretenden analizar los factores que pueden influir en la aparición de una fuga de cemento. En este sentido Ren et $\mathrm{al}^{39}$ pusieron de manifiesto que la pérdida de altura, el mayor volumen de cemento inyectado y la incompetencia de la pared vertebral, ya sea por la propia fractura o por una disrupción del pedículo durante el procedimiento, aumentan el riesgo de extravasación del cemento. Esta misma tendencia ha sido corroborada por otros autores como Chen et $\mathrm{al}^{8}$. En base a esto, se recomienda el uso de un volumen de cemento no superior a 4'17 mL, ya que, por otro lado, algunos estudios han demostrado que el resultado clínico del procedimiento no depende en absoluto del volumen de cemento inoculado ${ }^{20}$. Además, otros autores han destacado la importancia de una técnica quirúrgica exquisita bajo control radioscópico para preservar la integridad del pedículo ${ }^{27,34}$. Por otra parte, autores como Georgy, Lin et al o Nieuwenhuijse et $\mathrm{al}^{17,24,32}$ han informado acerca del papel fundamental que juega la viscosidad del cemento no sólo en la aparición de fugas, sino también en la distribución del cemento dentro de la vértebra, lo cual afecta a su efecto estabilizador. Así se ha observado que cuanto menor es la viscosidad del cemento en el momento de la inoculación mayor es el riesgo de extravasación del mismo. La radioopacidad inicial de los cementos biológicos debería tenerse también en cuenta.

Un aspecto que llama poderosamente nuestra atención es la evolución en el tiempo del material de refuerzo vertebral. Existen en la literatura gran cantidad de artículos en los que se pone de manifiesto que tanto la HA como otras sales de fosfato cálcico se reabsorben y son sustituidas gradualmente por tejido óseo ${ }^{10,12,38,41}$. Con objeto de evaluar la evolución en el tiempo del cemento usado en nuestro caso, comparamos retrospectivamente los exámenes realizados al paciente con fechas 16/06/2008, 18/12/2008 y 21/09/2010. Dichos estudios se llevaron a cabo mediante un escáner PHILIPS Brilliance 16, realizando planos axiales de $3 \mathrm{~mm}$ de espesor en los dos primeros y de $5 \mathrm{~mm}$ en el tercero. Se obtuvieron reconstrucciones sagitales de 1'5 mm de espesor en los tres estudios.

En el examen inicial (figura 1) se observan los dos aportes de cemento desde los pedículos y una fuga de material que llega al espacio epidural siguiendo el agujero nutricio vertebral. En la zona más radioopaca, la densidad del cemento supera ligeramente las 1700 unidades Hounsfield (UH), sobrepasando ampliamente la densidad de la cortical ósea vertebral (1100-1200 UH). En el examen de diciembre de 2008 (figura 2a) la densidad del cemento se ha reducido muy ligeramente, no alcanzando en ningún punto las 1700 UH. El cemento extravasado había disminuido muy discretamente su volumen. Finalmente, en el estudio de septiembre de 2010 (figura 2b), la densidad de la zona más radioopaca no llega a las $1600 \mathrm{UH}$ y el volumen de cemento en el espacio epidural ha disminuido de manera muy significativa.

La variación de las UH del cemento a lo largo del tiempo parece confirmar el hecho de que el cemento es sustituido progresivamente por tejido óseo. Sin embargo, ¿no debería haberse sustituido todo el cemento después de 2 años de evolución? Si dicha reabsorción ya se ha producido, ¿por qué sigue manteniéndose en la vértebra una densidad tan elevada? Aunque no hemos encontrado estudios que lo avalen, pensamos que parte de este fenómeno podría 
deberse a la naturaleza del material radioopaco que se usa en este tipo de cemento: carbonato de estroncio (tabla 4). El estroncio es un elemento químico que tiene una estructura atómica muy similar a la del calcio, lo cual hace que pueda incorporarse al hueso en formación en sustitución de este último. De hecho, esta característica se ha usado en otros campos de la medicina como la patología forense para el reconocimiento de restos óseos ${ }^{6}$. Nuestra suposición es que dicho estroncio se incorpora al hueso, manteniéndose así la radioopacidad de la vértebra a pesar de la reabsorción del cemento. Sin embargo, serían necesarios estudios al respecto para corroborar dicha hipótesis.

Querríamos también llamar la atención sobre el hecho de que, en el caso que nos ocupa, el cemento extravasado no está siendo sustituido por hueso, como ocurre con el que se halla dentro del compartimento vertebral, sino que, al parecer, está sufriendo un proceso de reabsorción controlada. Todos los estudios que hemos encontrado en la literatura ${ }^{10,12,38,41}$ tratan sobre la evolución del material de refuerzo dentro de un ambiente óseo, pero no hacen referencia a cómo evolucionaría el mismo fuera del espacio vertebral. Dado que la fuga de cemento mantiene continuidad con el interior de la vértebra, cabría pensar que podría producirse una osificación de la misma que progresase desde el punto de fuga hacia el exterior o bien el cemento extravasado podría provocar una osificación ectópica aun cuando perdiese contacto con el interior vertebral, aunque nosotros no hemos observado estos comportamientos. Serán necesarios estudios más amplios para aclarar estas cuestiones.

Finalmente, cabría preguntarnos cuándo debemos utilizar un cemento biológico en vez de uno acrílico. Como ya hemos expuesto anteriormente el principal beneficio de los cementos biológicos es su capacidad de osteointegración y su potencial de reabsorción y sustitución por hueso sano. Para que este proceso ocurra, es evidente que debemos partir de un hueso con buena capacidad de regeneración, es decir, un hueso sano y, sobre todo, joven. Por ello, la respuesta teórica a este interrogante sería suponer que los cementos biológicos estarán indicados, en principio, en pacientes jóvenes sin patología subyacente en la vértebra fracturada.

Sin embargo, en nuestra práctica clínica diaria hemos comprobado que esto no es del todo cierto, ya que existen pacientes jóvenes con fracturas traumáticas cuyas vértebras se comportan durante el remodelado vertebral como las de pacientes ancianos con osteoporosis, cuya estructura trabecular ofrece escasa resistencia a la distensión del balón de cifoplastia. Este aspecto fue comunicado por nuestro grupo bajo el epígrafe de compliance vertebral/interacción trabécula-balón ${ }^{26}$.

En los pacientes con osteoporosis la arquitectura vertebral está alterada, lo cual hace que la resistencia a ser expandida sea baja. Así, aunque nosotros introduzcamos un volumen determinado de contraste dentro del balón, la presión dentro del mismo se mantendrá baja, puesto que el soma vertebral tiende a expandirse sin ofrecer apenas resistencia.

Por el contrario, en pacientes jóvenes sin patología vertebral subyacente, ante pequeños aumentos de volumen en el balón de CP, se producen incrementos muy significativos de la presión, hasta alcanzar un determinado punto (punto de rotura), en el que la presión cae drásticamente y la vértebra comienza a expandirse. Además, es muy significativo el hecho de que cuanto más tiempo haya transcurrido entre la fractura y el tratamiento, mayor volumen y presión se precisa para alcanzar dicho punto de rotura, probablemente por una consolidación parcial de la fractura ${ }^{5,26}$.

Sin embargo, hemos podido comprobar que en muchos pacientes jóvenes con fracturas traumáticas el hueso se comporta como en pacientes con escasa resistencia a la distensión del balón, por lo cual pensamos que en estos casos puede subyacer una debilidad estructural de la vértebra que no se había manifestado previamente (no es el caso del paciente que presentamos, ya que en éste la densitometría ósea era normal). Por todo ello, en Nuestro Servicio se realiza el tratamiento de la forma más precoz posible y la elección del cemento biológico se basa fundamentalmente en la resistencia que observemos al hinchado del balón durante el procedimiento quirúrgico, aconsejando su uso sólo en pacientes con alta resistencia inicial a la distensión del balón.

\section{Conclusiones}

Los procedimientos de refuerzo vertebral son técnicas mínimamente invasivas indicadas en el tratamiento de fracturas vertebrales no sólo de origen osteoporótico o patológico, sino también en casos seleccionados de fracturas traumáticas. A pesar de ello son técnicas que no están exentas de complicaciones, destacando entre ellas la extravasación del cemento fuera del cuerpo vertebral.

Las principales medidas para evitar la fuga de cemento son la cuidadosa elección de los casos, una meticulosa técnica quirúrgica bajo control radioscópico y un volumen total de cemento no superior a 4 c.c.

Ambas técnicas pueden realizarse mediante cementos acrílicos o biológicos. Los biológicos tienen la ventaja de ser biocompatibles, biodegradables y osteoconductivos. Además, son los únicos que podrían sufrir fenómenos de reabsorción en caso de fuga. La elección de un tipo u otro dependerá de las características del paciente y, sobre todo, del tipo de interacción trabécula-balón.

Son necesarios estudios más amplios para terminar de aclarar el comportamiento de estos cementos a largo plazo y el papel que juega cada uno de sus componentes en el 
mismo, tanto dentro como fuera del compartimento óseo.

\section{Bibliografía}

1. Albee, F., Morrison, H.: Studies in bone growth: triple calcium phosphate as a stimulus to osteogenesis. Ann Surg 1920; 71: 32-38.

2. Anselmetti, G.C., Corrao, G., Monica, P.D., et al.: Pain relief following percutaneous vertebroplasty: results of a series of 283 consecutive patients treated in a single institution. Cardiovasc Intervent Radiol 2007; 30: 441-447.

3. Anselmetti, G.C., Muto, M., Guglielmi, G., Masala, S.: Percutaneous vertebroplasty or kyphoplasty. Radiol Clin N Am 2010; 48: 641-649.

4. Arregui, R., Martínez-Quiñones, J.V., Aso, J.: Fracturas dorsolumbares por flexo-compresión. Nuevas estrategias terapéuticas. IV Congreso de la Sociedad Española de Neurorraquis. Tenerife. 17-19 Febrero, 2004.

5. Arregui, R., Martínez-Quiñones, J.V., Aso-Escario, J., Aso-Vizán, J.: Papel del refuerzo vertebral mediante cifoplastia en el tratamiento de las fracturas dorsolumbares de índole no osteoporótico. Revisión del tema y análisis de 40 casos. Neurocirugía 2008; 19: 537-550.

6. Beard, B.L., Johnson, C.M.: Strontium isotope composition of skeletal material can determine the birth place and geographic mobility of humans and animals. J Forensic Sci 2000; 45: 1049-1061.

7. Charnley, J.: The bonding of prostheses to bone by cement. J Bone Joint Surg Br 1964; 46: 518-529.

8. Chen, W.J., Kao, Y.H., Yang, S.C., Yu, S.W., Tu, Y.K., Chung, K.C.: Impact of cement leakage into disks on the development of adjacent vertebral compression fractures. J Spinal Disord Tech 2010; 23: 35-39.

9. Chiras, J.: Percutaneous vertebral surgery: techniques and indications. J Neuroradiol 1997; 24: 45-52.

10. Chow, L.C.: Next generation calcium phosphate-based biomaterials. Dent Mater J 2009; 28: 1-10.

11. Cortet, B., Cotten, A., Boutry, N., et al.: Percutaneous vertebroplasty in the treatment of osteoporotic vertebral compression fractures: an open prospective study. J Rheumatol 1999; 26: 2222-2228.

12. Costantino, P.D., Friedman C.D., Jones, K., Chow, L.C., Sisson, G.A.: Experimental Hydroxyapatite cement. Cranioplasty Plast Reconstr Surg 1992; 90: 174-191.

13. Cotten, A., Dewatre, F., Cortet, B., et al.: Percutaneous vertebroplasty for osteolytic metastases and myeloma: effects of the percentage of lesion filling and the leakage of methylmethacrylate at clinical follow-up. Radiology 1996; 200: 525530 .

14. Deramond, H.: Percutaneous vertebroplasty with polymethylmethacrylate: technique, indication and results. Radiol Clin North Am 1988; 36: 533-546.

15. Galibert, P., Deramond, H., Rosat, P., Le Gars, D., et al.: Note préliminaire sur le traitment des angiomes vértebraux par vertébroplastie acrylique percutaneé. Neurochirurgie 1987; 33: 166-168.

16. Gangi, A., Guth, S., Imbert, J.P., et al.: Percutaneous vertebroplasty: indications, technique and results. Radiographics 2003; 23: e10.

17. Georgy, B.A.: Clinical experience with high-viscosity cements for percutaneous vertebral body augmentation: occurrence, degree and location of cement leakage compared with kyphoplasty. Am J Neuroradiol 2010; 31: 504-508.

18. Heini, P.F., Wälchli, B., Berlemann, U.: Percutaneous transpedicular vertebroplasty with PMMA: operative technique and early results. Eur Spine J 2000; 9: 445-450.

19. Hierholzer, J., Fuchs, H., Westphalen, K., et al.: Incidence of symptomatic vertebral fractures in patients after percutaneous vertebroplasty. Cardiovasc Intervent Radiol 2008; 31: 1178-1183.

20. Hulme, P.A., Krebs, J., Ferguson, S.J., et al.: Vertebroplasty and kyphoplasty: a systematic review of 69 clinical studies. Spine 2006; 31: 1983-2001.

21. Jaeblon, T.: Polymethylmethacrylate: Properties and Contemporary Uses in Orthopaedics. J Am Acad Orthop Surg 2010; 18: 297-305.

22. Jensen, M.E., Evans, A.J., Mathis, J.M., et al.: Percutaneous polymethylmethacrylate vertebroplasty in the treatmen of osteoporotic vertebral body compression fractures: technical aspects. AJNR Am J Neuroradiol 1997; 18: 1897-1904.

23. Lee, I.J., Choi, A.L., Yie, M.Y., et al.: CT evaluation of local leakage of bone cement after percutaneous kyphoplasty and vertebroplasty. Acta Radiol 2006; 6: 649-654.

24. Lin, S.W., Chiang, C.K., Yang, C.L., Wang, J.L.: Feasibility study of using viscoplastic bone cement for vertebroplasty. An in vivo clinical trial and in vitro cadaveric biomechanical examination. Spine 2010; 35: e385-e391.

25. Markovic, M., Takagi, S., Chow, L.C.: Formation of macropores in calcium phosphate cement through the use of mannitol crystals. Key Engineering Materiasl 2001; 195: 773776.

26. Martínez-Quiñones, J.V., Aso, J., Arregui, R.: Compliance vertebral. Su utilidad en el diagnóstico/tratamiento de las fracturas vertebrales agudas. XXII Congreso Nacional de la Sociedad GEER. Zaragoza, 6 y 7 de Junio de 2008.

27. Martínez-Quiñones, J.V., Aso-Escario, J., ArreguiCalvo, R.: Refuerzo vertebral percutáneo: vertebroplastia y cifoplastia: Procedimiento técnico. Neurocirugía 2005; 16 : 427-440.

28. Martínez-Quiñones, J.V., Hernández-Sánchez, G.: La vertebroplastia percutánea: Técnica y resultados preliminares en 25 procedimientos. Neurocirugía 2003; 14: 323-332.

29. Masala, S., Mammucari, M., Angelopoulos, G., et al.: Percutaneous vertebroplasty in the Management of vertebral osteoporotic fractures. Short-term, mid-term and long-term follow-up of 285 patients. Skeletal Radiol 2009; 38: 863-869. 
30. Mcgirt, M.J., Parker, S.L., Wolinsky, J.P., et al.: Vertebroplasty and kyphoplasty for the treatment of vertebral compression fractures: an evidenced-based review of the literature. Spine J 2009; 9: 501-508.

31. Meraw, S.J., Reeve, C.M., Lohse, C.M., Sioussat, T.M.: Treatment of Peri-Implant defects with Combination Growth Factor Cements. J Periodont 2000; 71: 8-13.

32. Nieuwenhuijse, M.J., Muijs, S.P.J., van Erkel, A.R., Dijkstra, S.P.D.: A clinical comparative study on low versus medium viscosity polymethylmetacrylate bone cement in percutaneous vertebroplasty. Viscosity associated with cement leakage. Spine 2010; 35: e1037-e1044.

33. Ofluoglu, O.: Minimally invasive management of spinal metastases. Orthop Clin North Am 2009; 40: 155-168.

34. Park, S.Y., Modi, H.N., Suh, S.W., Hong, J.Y., Noh, W., Yang, J.H.: Epidural cement leakage through pedicle violation after ballon kyphoplasty causing paraparesis in osteoporotic vertebral compression fractures - a report of two cases. Journal of Orthopaedic Surgery and Research 2010; 5: 54.

35. Phillips, F.M., Pfeifer, B.A., Lieberman, I.H., et al.: Minimally invasive treatments of osteoporotic vertebral compression fractures: vertebroplasty and kyphoplasty. Instr Course Lect 2003; S2: 559-567.

36. Phillips, F.M., Todd-Wetzel, F., Lieberman, I., Campbell-Hupp, M.: An in vivo comparison of the potential for extravertebral cement leak after vertebroplasty and kyphoplasty. Spine 2002; 27: 2173-2178.

37. Ploeg, W.T., Veldhuizen, A.G., The, B., et al.: Percutaneous vertebroplasty as a treatment for osteoporotic vertebral compression fractures: systematic review. Eur Spine J 2006; 15: 1749-1758.

38. Ray, R.D., Ward, A.A.: A preliminary report on studies of basic calcium phosphate in bone replacement. Surgical Forum, American College of Surgeons. Philadelphia. 1951.

39. Ren, H., Shen, Y., Zhang, Y.Z., et al.: Correlative factor analysis on the complications resulting from cement leakage after percutaneous kyphoplasty in the treatment of osteoporotic vertebral compression fracture. J Spinal Disord Tech 2010; 23: e9-e15.

40. Siemund, R., Nilsson, L.T., Cronquist, M., Strömquist, B.: Initial Clinical Experience with a new biointegrative cement for vertebroplasty in osteoporotic vertebral fractures. Interv Neuroradiol 2009; 15: 335-340.

41. Suzuki, O., Nakamura, M., Miyasaka, Y., Kagayama, M., Sakurai, M.: Bone formation on synthetic precursors of hydroxyapatite. Tohoku J Exp Med 1991; 164: 37-50.

42. Takagi, S., Chow, L.C.: Formation of macropores in calcium phosphate cement implants. J Mater Sci 2001; 12: 135-139.

43. Takagi, S., Chow, L.C., Hirayama, S., Sugawara, A.: Pemixed calcium phosphates cement pastes. J Biomed Mater Res Part B Biomater 2003; 67: 689-696.

44. Truumees, E., Hilibrand, A., Vaccaro, A.R.: Percutaneous vertebral augmentation. Spine J 2004; 4: 218-229.

45. Watts, N.B., Harris, S.T., Genant, H.K.: Treatment of painful osteoporotic vertebral fractures with percutaneous vertebroplasty or kyphoplasty. Osteoporos Int 2001; 12: 429437.

46. Weill, A., Chiras, J., Simon, et al.: Spinal metastases: indications for and results of percutaneous injection of acrylic surgical cement. Radiology 1996; 199: 241-247.

47. Xu, H.H., Quinn, J.B., Takagi, S., Chow, L.C.: Synergistic reinforcement of in situ hardening calcium phosphate composite scaffold for bone tissue engineering. Biomaterials 2004; 25: 1029-1037.

48. Yeom, J.S., Kim, W.J., Choy, W.S., Lee, C.K., Chang, B.S., Kang, J.W.: Leakage of cement in percutaneous transpedicular vertebroplasty for painful osteoporotic compression fractures. J Bone Joint Surg Br 2003; 85: 83-89.

49. Yu, S.W., Yang, S.C., Kao, Y.H., et al.: Clinical evaluation of vertebroplasty for multiple-level osteoporotic spinal compression fracture in the elderly. Arch Orthop Trauma Surg 2008; 128: 97-101.

Martínez-Quiñones, J.V.; Medina-Imbroda, J.M.; Aso-Escario, J.; Pons Bosque, J.; Consolini, F.E.; Arregui-Calvo, R.: Fuga intrarraquídea de cemento biológico tras cifoplastia. Evolución a largo plazo. Presentación de un caso y revisión de la literatura. Neurocirugía 2011; 22: 588-599.

Correspondencia: José Vicente Martinez Quiñones. jvmartinez@maz.es chevimq@gmail.com 